\title{
A QUASI-THETA PRODUCT IN RAMANUJAN'S LOST NOTEBOOK
}

\author{
BRUCE C. BERNDT ${ }^{1}$, HENG HUAT CHAN, AND ALEXANDRU ZAHARESCU
}

\begin{abstract}
On page 209 in his lost notebook, Ramanujan records an unusual product formula, reminiscent of a product of theta functions. The formula involves hypergeometric functions and has a connection with elliptic functions. In this paper, we prove the formula, offer some generalizations, and indicate some further connections with Ramanujan's work.
\end{abstract}

\section{INTRODUCTION}

At the top of page 209 in the Narosa edition of his lost notebook [5], Ramanujan recorded the enigmatic formula,

$$
\begin{aligned}
\left\{\prod_{n=0}^{\infty}\left(\frac{1-(-1)^{n} q^{(2 n+1) / 2}}{1+(-1)^{n} q^{(2 n+1) / 2}}\right)^{2 n+1}\right\}^{\log q} & \left\{\prod_{n=1}^{\infty}\left(\frac{1+(-1)^{n} i q^{\prime n}}{1-(-1)^{n} i q^{\prime n}}\right)^{n}\right\}^{2 \pi i} \\
& =\exp \left(\frac{\pi^{2}}{4}-\frac{k_{3} F_{2}\left(1,1,1 ; \frac{3}{2}, \frac{3}{2} ; k^{2}\right)}{{ }_{2} F_{1}\left(\frac{1}{2}, \frac{1}{2} ; 1 ; k^{2}\right)}\right),
\end{aligned}
$$

where

$$
q=\exp \left(-\pi K^{\prime} / K\right), \quad q^{\prime}=\exp \left(-\pi K / K^{\prime}\right), \quad \text { and } \quad 0<k<1 .
$$

Because of poor photocopying, (1.1) is very difficult to read in [5]. If the powers $2 n+1$ and $n$ on the two pairs of large parentheses were absent, the products could be expressed in terms of theta functions. Ramanujan did not use the notation ${ }_{3} F_{2}$ and ${ }_{2} F_{1}$ for hypergeometric functions, but instead only recorded the first three terms of each series. Also, Ramanujan did not divulge the meaning of the notations $K$ and $K^{\prime}$. However, from considerable work in both the ordinary notebooks [4] and lost notebook [5], we can easily deduce that $K$ denotes the complete elliptic integral of the first kind defined by

$$
K:=K(k):=\int_{0}^{\pi / 2} \frac{d \varphi}{\sqrt{1-k^{2} \sin ^{2} \varphi}},
$$

where $k, 0<k<1$, denotes the modulus. Furthermore, $K^{\prime}=K\left(k^{\prime}\right)$, where $k^{\prime}:=$ $\sqrt{1-k^{2}}$ is the complementary modulus.

There are no other formulas like (1.1) in Ramanujan's work, and apparently there are none like it in the literature as well. The purpose of this paper is to prove (1.1). As will be seen in our proof, the unique character of (1.1) derives from a single, almost

\footnotetext{
${ }^{1}$ Research partially supported by grant MDA904-00-1-0015 from the National Security Agency.

${ }^{2} 2000$ AMS Classification Numbers: Primary, 11F27; Secondary, 33D10, 33C05.
} 
miraculous, connection with the theory of elliptic functions given in the identity

$$
\sum_{n=0}^{\infty} \frac{1}{(2 n+1)^{2} \cosh \{(2 n+1) \alpha / 2\}}=\frac{k}{2 z}{ }_{3} F_{2}\left(1,1,1 ; \frac{3}{2}, \frac{3}{2} ; k^{2}\right)
$$

where $\alpha=\pi K^{\prime} / K$ and $z={ }_{2} F_{1}\left(\frac{1}{2}, \frac{1}{2} ; 1 ; k^{2}\right)$. The identity (1.3) is found in Entry 6 of Chapter 18 in Ramanujan's second notebook [4], [1, p. 153]. Like many of Ramanujan's discoveries, (1.3) is not fully understood. Is this connection between hypergeometric series and elliptic functions a singular accident, or are there deeper, still to be recognized connections? In his notebooks [4, p. 280], Ramanujan also attempted to find a formula similar to (1.3), but with $(2 n+1)^{2}$ replaced by $(2 n+1)^{4}$. In fact, Ramanujan struck out his imprecisely stated formula by putting two lines through it. See [2, pp. 397-403] for Berndt's failed attempt to find a correct version.

In Section 2, we first establish in Theorem 2.1 an equivalent formulation of (1.1) as an identity amongst infinite series of hyperbolic trigonometric functions. Secondly, we prove this identity.

In Section 3, we briefly indicate generalizations of (1.1) and Theorem 2.1 and offer some related hyperbolic series of Ramanujan.

\section{An Equivalent Formulation of (1.1) in Terms of Hyperbolic Series}

Theorem 2.1. Let $\alpha$ and $\beta$ be any complex numbers with nonzero real parts and with $\alpha \beta=\pi^{2}$. Then (1.1) is equivalent to the identity

$$
\begin{aligned}
\alpha \sum_{n=0}^{\infty} \frac{\sinh \{(2 n+1) \alpha / 2\}}{(2 n+1) \cosh ^{2}\{(2 n+1) \alpha / 2\}}+\pi \sum_{n=0}^{\infty} \frac{(-1)^{n}}{(2 n+1) \cosh ^{2}\{(2 n+1) \beta / 2\}} \\
=\frac{\pi^{2}}{4}-2 \sum_{n=0}^{\infty} \frac{1}{(2 n+1)^{2} \cosh \{(2 n+1) \alpha / 2\}} .
\end{aligned}
$$

Proof. We assume that $\alpha$ and $\beta$ are positive real numbers. The general result will then follow by analytic continuation. Taking logarithms on both sides of (1.1), we find that

$$
\begin{gathered}
\log \left(\left\{\prod_{n=0}^{\infty}\left(\frac{1-(-1)^{n} q^{(2 n+1) / 2}}{1+(-1)^{n} q^{(2 n+1) / 2}}\right)^{2 n+1}\right\}^{\log q}\right)+\log \left(\left\{\prod_{n=1}^{\infty}\left(\frac{1+(-1)^{n} i q^{\prime n}}{1-(-1)^{n} i q^{\prime n}}\right)^{n}\right\}^{2 \pi i}\right) \\
=\frac{\pi^{2}}{4}-\frac{k_{3} F_{2}\left(1,1,1 ; \frac{3}{2}, \frac{3}{2} ; k^{2}\right)}{{ }_{2} F_{1}\left(\frac{1}{2}, \frac{1}{2} ; 1 ; k^{2}\right)}
\end{gathered}
$$

(Here and in the following step, we have ignored branches of the logarithm. The justification lies in our eventual proof of (2.1).) For brevity, let $L$ and $R$ denote, 
respectively, the left and right sides of (2.2). Then

$$
\begin{aligned}
L= & \log q\left(\sum_{n=0}^{\infty}(2 n+1)\left\{\log \left(1-(-1)^{n} q^{(2 n+1) / 2}\right)-\log \left(1+(-1)^{n} q^{(2 n+1) / 2}\right)\right\}\right) \\
& +2 \pi i\left(\sum_{n=1}^{\infty} n\left\{\log \left(1+(-1)^{n} i q^{\prime n}\right)-\log \left(1-(-1)^{n} i q^{\prime n}\right)\right\}\right) \\
= & : \log q\left(S_{1}-S_{2}\right)+2 \pi i\left(S_{3}-S_{4}\right) .
\end{aligned}
$$

Recall that $q$ and $q^{\prime}$ are defined in (1.2). Set $\alpha=\pi K^{\prime} / K$ and $\beta=\pi K / K^{\prime}$, so that $\alpha \beta=\pi^{2}$. We now proceed to show that $S_{1}, \ldots, S_{4}$ can be expressed as sums of hyperbolic functions.

Using the Taylor series of $\log (1+z)$ about $z=0$ and recalling the definition of $\beta$, we find that

$$
\begin{aligned}
S_{3} & =-\sum_{n=1}^{\infty} \sum_{m=1}^{\infty} n \frac{(-1)^{m+m n} i^{m} e^{-\beta m n}}{m} \\
& =-\sum_{m=1}^{\infty} \frac{(-i)^{m}}{m} \sum_{n=1}^{\infty} n\left\{\left(-e^{-\beta}\right)^{m}\right\}^{n} \\
& =-\sum_{m=1}^{\infty} \frac{\left(i e^{-\beta}\right)^{m}}{m\left(1-\left(-e^{-\beta}\right)^{m}\right)^{2}} .
\end{aligned}
$$

By a similar calculation,

$$
S_{4}=-\sum_{m=1}^{\infty} \frac{\left(-i e^{-\beta}\right)^{m}}{m\left(1-\left(-e^{-\beta}\right)^{m}\right)^{2}} .
$$

Combining (2.4) and (2.5), we find that

$$
\begin{aligned}
S_{3}-S_{4} & =\sum_{m=1}^{\infty} \frac{-\left(i e^{-\beta}\right)^{m}+\left(-i e^{-\beta}\right)^{m}}{m\left(1-\left(-e^{-\beta}\right)^{m}\right)^{2}} \\
& =-2 i \sum_{m=0}^{\infty} \frac{(-1)^{m} e^{-(2 m+1) \beta}}{(2 m+1)\left(1+e^{-(2 m+1) \beta}\right)^{2}} \\
& =-\frac{i}{2} \sum_{m=0}^{\infty} \frac{(-1)^{m}}{(2 m+1) \cosh ^{2}\{(2 m+1) \beta / 2\}} .
\end{aligned}
$$


Next, again using the Taylor series of $\log (1+z)$ about $z=0$ and recalling the definition of $\alpha$, we find that

$$
\begin{aligned}
S_{1} & =-\sum_{n=0}^{\infty} \sum_{m=1}^{\infty}(2 n+1) \frac{(-1)^{m n} e^{-\alpha(2 n+1) m / 2}}{m} \\
& =-\sum_{m=1}^{\infty} \frac{1}{m}\left(\sum_{n=0}^{\infty}\left(2 n(-1)^{m n} e^{-\alpha(2 n+1) m / 2}+(-1)^{m n} e^{-\alpha(2 n+1) m / 2}\right)\right) \\
& =-\sum_{m=1}^{\infty} \frac{1}{m}\left(\frac{2(-1)^{m} e^{-3 \alpha m / 2}}{\left(1-\left(-e^{-\alpha}\right)^{m}\right)^{2}}+\frac{e^{-\alpha m / 2}}{1-\left(-e^{-\alpha}\right)^{m}}\right) \\
& =-\sum_{m=1}^{\infty} \frac{(-1)^{m} e^{-3 \alpha m / 2}+e^{-\alpha m / 2}}{m\left(1-\left(-e^{-\alpha}\right)^{m}\right)^{2}} .
\end{aligned}
$$

By an analogous argument,

$$
S_{2}=-\sum_{m=1}^{\infty} \frac{e^{-3 \alpha m / 2}+(-1)^{m} e^{-\alpha m / 2}}{m\left(1-\left(-e^{-\alpha}\right)^{m}\right)^{2}} .
$$

Thus, combining (2.7) and (2.8), we deduce that

$$
\begin{aligned}
S_{1}-S_{2} & =\sum_{m=1}^{\infty} \frac{-(-1)^{m} e^{-3 \alpha m / 2}-e^{-\alpha m / 2}+e^{-3 \alpha m / 2}+(-1)^{m} e^{-\alpha m / 2}}{m\left(1-\left(-e^{-\alpha}\right)^{m}\right)^{2}} \\
& =2 \sum_{m=0}^{\infty} \frac{e^{-3(2 m+1) \alpha / 2}-e^{-(2 m+1) \alpha / 2}}{(2 m+1)\left(1+e^{-(2 m+1) \alpha}\right)^{2}} \\
& =2 \sum_{m=0}^{\infty} \frac{e^{-(2 m+1) \alpha / 2}-e^{(2 m+1) \alpha / 2}}{(2 m+1)\left(e^{(2 m+1) \alpha / 2}+e^{-(2 m+1) \alpha / 2}\right)^{2}} \\
& =-\sum_{m=0}^{\infty} \frac{\sinh \{(2 m+1) \alpha / 2\}}{(2 m+1) \cosh ^{2}\{(2 m+1) \alpha / 2\}} .
\end{aligned}
$$

If we use (2.6) and (2.9) in (2.3) and recall that $\log q=-\alpha$, we deduce that

$$
\begin{aligned}
\alpha \sum_{m=0}^{\infty} \frac{\sinh \{(2 m+1) \alpha / 2\}}{(2 m+1) \cosh ^{2}\{(2 m+1) \alpha / 2\}} & +\pi \sum_{m=0}^{\infty} \frac{(-1)^{m}}{(2 m+1) \cosh ^{2}\{(2 m+1) \beta / 2\}} \\
= & \frac{\pi^{2}}{4}-\frac{k_{3} F_{2}\left(1,1,1 ; \frac{3}{2}, \frac{3}{2} ; k^{2}\right)}{{ }_{2} F_{1}\left(\frac{1}{2}, \frac{1}{2} ; 1 ; k^{2}\right)} .
\end{aligned}
$$

We now invoke (1.3). If we substitute (1.3) into (2.10), we deduce (2.1) to complete the proof.

It should be emphasized that the only time we used the definitions (1.2) of $q$ and $q^{\prime}$ in our proof is in the application of (1.3). Thus, it would seem that (1.1) is a very special result in that there are likely very few (if any) other results like it.

We now prove (2.1). 
Proof of (2.1). Our first main idea is to introduce the functions $F$ and $G$ in (2.11) and (2.13), respectively, and use them to find a simpler identity which is equivalent to (2.1). Define

$$
F(\alpha):=\frac{1}{\alpha} \sum_{n=0}^{\infty} \frac{1}{(2 n+1)^{2} \cosh \{(2 n+1) \alpha / 2\}}
$$

Then

$$
\begin{aligned}
F^{\prime}(\alpha):= & -\frac{1}{2 \alpha^{2}}\left(\alpha \sum_{n=0}^{\infty} \frac{\sinh \{(2 n+1) \alpha / 2\}}{(2 n+1) \cosh ^{2}\{(2 n+1) \alpha / 2\}}\right. \\
& \left.+2 \sum_{n=0}^{\infty} \frac{1}{(2 n+1)^{2} \cosh \{(2 n+1) \alpha / 2\}}\right) .
\end{aligned}
$$

Set

$$
G(\beta):=2 \beta \sum_{n=0}^{\infty} \frac{1}{(2 n+1)^{2} \cosh \left\{(2 n+1) \pi^{2} /(2 \beta)\right\}}=2 \pi^{2} F\left(\frac{\pi^{2}}{\beta}\right),
$$

by (2.11) and the fact that $\alpha \beta=\pi^{2}$. Thus, by (2.12),

$$
\begin{aligned}
G^{\prime}(\beta)=2 \pi^{2} F^{\prime}\left(\frac{\pi^{2}}{\beta}\right)\left(-\frac{\pi^{2}}{\beta^{2}}\right)= & \alpha \sum_{n=0}^{\infty} \frac{\sinh \left\{(2 n+1) \pi^{2} /(2 \beta)\right\}}{(2 n+1) \cosh ^{2}\left\{(2 n+1) \pi^{2} /(2 \beta)\right\}} \\
& +2 \sum_{n=0}^{\infty} \frac{1}{(2 n+1)^{2} \cosh \left\{(2 n+1) \pi^{2} /(2 \beta)\right\}}
\end{aligned}
$$

If we define

$$
H(\beta):=\frac{\beta \pi^{2}}{4}-2 \pi \sum_{n=0}^{\infty} \frac{(-1)^{n} \tanh \{(2 n+1) \beta / 2\}}{(2 n+1)^{2}}
$$

then

$$
H^{\prime}(\beta):=\frac{\pi^{2}}{4}-\pi \sum_{n=0}^{\infty} \frac{(-1)^{n}}{(2 n+1) \cosh ^{2}\{(2 n+1) \beta / 2\}} .
$$

In view of (2.14) and (2.16), we see that (2.1) is equivalent to

$$
G^{\prime}(\beta)=H^{\prime}(\beta) \text {. }
$$

It follows that for some constant $c$,

$$
G(\beta)=H(\beta)+c .
$$

Clearly, from the definitions of $G(\beta)$ and $H(\beta)$ in (2.13) and (2.15), respectively, both $G(\beta)$ and $H(\beta)$ tend to 0 as $\beta \rightarrow 0$. Thus, in $(2.17), c=0$.

Hence, it now suffices to prove that

$$
2 \beta \sum_{n=0}^{\infty} \frac{1}{(2 n+1)^{2} \cosh \left\{(2 n+1) \pi^{2} /(2 \beta)\right\}}=\frac{\beta \pi^{2}}{4}-2 \pi \sum_{n=0}^{\infty} \frac{(-1)^{n} \tanh \{(2 n+1) \beta / 2\}}{(2 n+1)^{2}} .
$$


It is easily seen that $(2.18)$ is equivalent to

$\beta \sum_{n=-\infty}^{\infty} \frac{1}{(2 n+1)^{2} \cosh \left\{(2 n+1) \pi^{2} /(2 \beta)\right\}}+\pi \sum_{n=-\infty}^{\infty} \frac{(-1)^{n} \tanh \{(2 n+1) \beta / 2\}}{(2 n+1)^{2}}-\frac{\beta \pi^{2}}{4}=0$.

The second primary idea is to introduce a function $f$ of a complex variable and use contour integration to prove (2.19). To that end, define, for fixed $\eta>0$,

$$
f(z):=\frac{\tan (\eta z)}{z^{2} \cosh z}
$$

The function $f(z)$ is meromorphic in the entire complex plane with a simple pole at $z=0$ and simple poles at $z=(2 n+1) \pi i / 2$ and $z=(2 n+1) \pi /(2 \eta)$ for each integer $n$. Let $\gamma_{R_{m}}$ be a sequence of positively oriented circles centered at the origin and with radii $R_{m}$ tending to $\infty$ as $m \rightarrow \infty$, where the radii $R_{m}$ are chosen so that the circles remain at a bounded distance from all the poles of $f(z)$. From the definition (2.20) of $f$, it is then easy to see that

$$
\left|\int_{\gamma_{R_{m}}} f(z) d z\right| \ll_{\eta} \frac{1}{R_{m}}
$$

as $R_{m} \rightarrow \infty$.

For brevity, let $R(a)$ denote the residue of $f(z)$ at a pole $a$. Then, brief calculations show that

$$
\begin{aligned}
R(0) & =\eta \\
R\left(\frac{(2 n+1) \pi}{2 \eta}\right) & =-\frac{4 \eta}{\pi^{2}(2 n+1)^{2} \cosh \{(2 n+1) \pi /(2 \eta)\}}, \\
R\left(\frac{(2 n+1) \pi i}{2}\right) & =-\frac{4(-1)^{n} \tanh \{(2 n+1) \pi \eta / 2\}}{\pi^{2}(2 n+1)^{2}},
\end{aligned}
$$

for each integer $n$. Hence, using (2.22)-(2.24) and the residue theorem, we deduce that

$$
\begin{aligned}
\frac{1}{2 \pi i} \int_{\gamma_{R m}} f(z) d z= & -\sum_{|2 n+1|<2 \eta R_{m} / \pi} \frac{4 \eta}{\pi^{2}(2 n+1)^{2} \cosh \{(2 n+1) \pi /(2 \eta)\}} \\
& -\sum_{|2 n+1|<2 R_{m} / \pi} \frac{4(-1)^{n} \tanh \{(2 n+1) \pi \eta / 2\}}{\pi^{2}(2 n+1)^{2}} .
\end{aligned}
$$

Letting $R_{m}$ tend to $\infty$ in (2.25) and employing (2.21), we conclude that

$0=\eta-\frac{4 \eta}{\pi^{2}} \sum_{n=-\infty}^{\infty} \frac{1}{(2 n+1)^{2} \cosh \{(2 n+1) \pi /(2 \eta)\}}-\frac{4}{\pi^{2}} \sum_{n=-\infty}^{\infty} \frac{(-1)^{n} \tanh \{(2 n+1) \pi \eta / 2\}}{(2 n+1)^{2}}$.

Now set $\eta=\beta / \pi$ in $(2.26)$. Then multiply both sides by $-\pi^{3} / 4$. We then readily obtain (2.19), and so this completes the proof. 


\section{Concluding Remarks}

Theorem 2.1 can easily be generalized in at least two directions.

First, in the proof of (2.1), we could replace $f(z)$ by

$$
f_{n}(z):=\frac{\tan (\eta z)}{z^{n} \cosh z}
$$

where $n$ is a positive integer exceeding 1. The generalization of (2.1) would then involve Bernoulli numbers arising from the Taylor expansion of tan $z$ about $z=0$ and Euler numbers arising from the expansion of $1 / \cosh z$ about $z=0$.

Second, in the proof of (2.1), we could replace $f(z)$ by

$$
f(z, \theta):=\frac{\cosh (\theta z) \tan (\eta z)}{z^{2} \cosh z}
$$

where $-1<\theta<1$. Then by a proof analogous to that given above, we can deduce that, for any complex numbers $\alpha$ and $\beta$ with $\operatorname{Re} \alpha$, $\operatorname{Re} \beta \neq 0$ and $\alpha \beta=\pi^{2}$, and for any real number $\theta$ with $|\theta|<1$,

$$
\begin{aligned}
& \alpha \sum_{n=0}^{\infty} \frac{\sinh \{(2 n+1) \alpha / 2\} \cosh \{(2 n+1) \theta \alpha / 2\}}{(2 n+1) \cosh ^{2}\{(2 n+1) \alpha / 2\}}-\theta \alpha \sum_{n=0}^{\infty} \frac{\sinh \{(2 n+1) \theta \alpha / 2\}}{(2 n+1) \cosh \{(2 n+1) \alpha / 2\}} \\
+ & \pi \sum_{n=0}^{\infty} \frac{(-1)^{n} \cos \{(2 n+1) \pi \theta / 2\}}{(2 n+1) \cosh ^{2}\{(2 n+1) \beta / 2\}}=\frac{\pi^{2}}{4}-2 \sum_{n=0}^{\infty} \frac{\cosh \{(2 n+1) \theta \alpha / 2\}}{(2 n+1)^{2} \cosh \{(2 n+1) \alpha / 2\}} .
\end{aligned}
$$

The identity (3.1) is equivalent to

$$
\begin{aligned}
& \quad\left\{\prod_{n=0}^{\infty}\left(\frac{\left(1-(-1)^{n} q^{(2 n+1-\theta) / 2}\right)\left(1-(-1)^{n} q^{(2 n+1+\theta) / 2}\right)}{\left(1+(-1)^{n} q^{(2 n+1-\theta) / 2}\right)\left(1+(-1)^{n} q^{(2 n+1+\theta) / 2}\right)}\right)^{2 n+1}\right\}^{(\log q) / 2} \\
& \times\left\{\prod_{n=0}^{\infty} \frac{\left(1-(-1)^{n} q^{(2 n+1-\theta) / 2}\right)\left(1+(-1)^{n} q^{(2 n+1+\theta) / 2}\right)}{\left(1+(-1)^{n} q^{(2 n+1-\theta) / 2}\right)\left(1-(-1)^{n} q^{(2 n+1+\theta) / 2}\right)}\right\}^{-\theta(\log q) / 2} \\
& \times\left\{\prod_{n=1}^{\infty}\left(\frac{\left(1+(-1)^{n} i e^{\theta \pi i / 2} q^{\prime n}\right)\left(1+(-1)^{n} i e^{-\theta \pi i / 2} q^{\prime n}\right)}{\left(1-(-1)^{n} i e^{\theta \pi i / 2} q^{\prime n}\right)\left(1-(-1)^{n} i e^{-\theta \pi i / 2} q^{\prime n}\right)}\right)^{n}\right\}^{\pi i} \\
& =\exp \left(\frac{\pi^{2}}{4}-2 \sum_{n=0}^{\infty} \frac{\cosh \{(2 n+1) \theta \alpha / 2\}}{(2 n+1)^{2} \cosh \{(2 n+1) \alpha / 2\}}\right)
\end{aligned}
$$

When $\theta=0,(3.1)$ and (3.2) reduce to (2.1) and (1.1), respectively. If $\alpha, \beta>0$ and $\theta=: u+i v$, where $u$ and $v$ are real, then (3.2) can be analytically continued to the rectangle $-1<u<1,-2 \pi / \alpha<v<2 \pi / \alpha$.

If we differentiate (3.1) $2 k$ times with respect to $\theta$ and then set $\theta=0$ we deduce that 


$$
\begin{aligned}
\alpha \sum_{n=0}^{\infty} \frac{(2 n+1)^{2 k-1} \sinh \{(2 n+1) \alpha / 2\}}{\cosh ^{2}\{(2 n+1) \alpha / 2\}} & +(-1)^{k} \beta^{2 k} \pi^{1-2 k} \sum_{n=0}^{\infty} \frac{(-1)^{n}(2 n+1)^{2 k-1}}{\cosh ^{2}\{(2 n+1) \beta / 2\}} \\
= & (4 k-2) \sum_{n=0}^{\infty} \frac{(2 n+1)^{2 k-2}}{\cosh \{(2 n+1) \alpha / 2\}},
\end{aligned}
$$

which is valid for any integer $k \geq 1$ and any complex numbers $\alpha$ and $\beta$ with $\operatorname{Re} \alpha, \operatorname{Re} \beta \neq$ 0 and $\alpha \beta=\pi^{2}$.

If we let $\alpha \rightarrow \infty$ (or $\beta \rightarrow 0$ ) in (2.1), we deduce Leibniz's well-known evaluation

$$
\sum_{n=0}^{\infty} \frac{(-1)^{n}}{2 n+1}=\frac{\pi}{4}
$$

while if we let $\alpha \rightarrow 0$ (or $\beta \rightarrow \infty)$ in (2.1), we deduce Euler's well-known evaluation

$$
\sum_{n=0}^{\infty} \frac{1}{(2 n+1)^{2}}=\frac{\pi^{2}}{8}
$$

(We remark that care must be taken when taking certain limits inside summation signs above.)

Ramanujan examined several other infinite series of hyperbolic functions in his notebooks [4] and lost notebook [5]. We cite two examples giving evaluations of series involving $\cosh z$ which are very similar to those arising above.

First, in Entry 16(x) of Chapter 17 in his second notebook [4], [1, p. 134], Ramanujan asserted that

$$
\sum_{n=0}^{\infty} \frac{(2 n+1)^{2}}{\cosh \{(2 n+1) \pi / 2\}}=\frac{\pi^{3 / 2}}{2 \sqrt{2} \Gamma^{6}\left(\frac{3}{4}\right)} .
$$

In fact, it is shown in [1, pp. 134-138] that one can also evaluate in closed form the more general sum

$$
\sum_{n=0}^{\infty} \frac{(2 n+1)^{2 m}}{\cosh \{(2 n+1) \alpha / 2\}},
$$

where $m$ is a positive integer. However, the evaluations are in terms of $z:={ }_{2} F_{1}\left(\frac{1}{2}, \frac{1}{2} ; 1 ; k^{2}\right)$. (See [1, p. 101, eq. (6.3)] for the relation between $\alpha$ and $k$, where in [1], $y=\alpha$ ). Note that the sums (3.5) appear on the right side of (3.3), and so these evaluations also automatically yield evaluations for the left side of (3.3).

Second, the evaluation

$$
\sum_{n=0}^{\infty} \frac{(2 n+1)^{2}}{\cosh ^{2}\{(2 n+1) \pi / 2\}}=\frac{\pi^{2}}{12 \Gamma^{8}\left(\frac{3}{4}\right)}
$$

arises in Ramanujan's formulas for the power series coefficients of the reciprocals, or, more generally, quotients, of certain Eisenstein series [3, Cor. 3.9].

In a future paper, the authors will study a multi-variable generalization of the products in (1.1) and derive a transformation formula for them. 


\section{REFERENCES}

[1] B. C. Berndt. Ramanujan's Notebooks, Part III (Springer-Verlag, 1991).

[2] B. C. Berndt. Ramanujan's Notebooks, Part V (Springer-Verlag, 1998).

[3] B. C. Berndt, P. R. Bialek, and A. J. Yee. Formulas of Ramanujan for the power series coefficients of certain quotients of Eisenstein series. Internat. Math. Res. Not., to appear.

[4] S. Ramanujan. Notebooks (2 volumes) (Tata Institute of Fundamental Research, 1957).

[5] S. Ramanujan. The Lost Notebook and Other Unpublished Papers (Narosa, 1988).

Department of Mathematics, University of Illinois, 1409 West Green Street, UrBANA, IL 61801, USA

E-mail address: berndt@math.uiuc.edu

Department of Mathematics, National University of Singapore, Kent Ridge, SingaPORE 119260, RePUblic OF Singapore

E-mail address: chanhh@math.nus.edu.sg

Department of Mathematics, University of Illinois, 1409 West Green Street, UrBANA, IL 61801, USA

E-mail address: zaharesc@math.uiuc.edu 